\title{
The eclipsing binary star RZ Cas
}

\section{First spectroscopic detection of rapid pulsations in an Algol system ${ }^{\star}, \star \star$}

\author{
H. Lehmann ${ }^{1}$ and D. E. Mkrtichian ${ }^{2,3}$ \\ 1 Thüringer Landessternwarte, 07778 Tautenburg, Germany \\ 2 Astrophysical Research Center of the Structure and Evolution of the Cosmos, Sejong University, Seoul 143-747, Korea \\ e-mail: david@arcsec.sejong.ac.kr \\ 3 Astronomical Observatory, Odessa National University, Shevchenko Park, 65014, Odessa, Ukraine
}

Received 17 April 2003 / Accepted 19 September 2003

\begin{abstract}
In a first report on the results of a multi-site campaign in 2001 of photometric and spectroscopic observations of the active semi-detached Algol-type system RZ Cas, we concentrate on the radial velocity (RV) variations. Using weak absorption lines we obtain an improved orbital solution for both components. In the orbital RV curve we observe a strong, asymmetric rotation effect. For the first time we detect rapid spectroscopic multi-mode pulsations in an Algol system. Whereas the photometrically observed oscillations were dominated by monoperiodic pulsations at frequency $64.19 \mathrm{c} \mathrm{d}^{-1}$ until the year 2000 , we find in 2001 a multiperiodic behaviour with two dominant frequencies of $f_{1}=56.600 \mathrm{c} \mathrm{d}^{-1}$ and $f_{2}=64.189 \mathrm{c} \mathrm{d}^{-1}$.

Both modes show amplitude variations over the orbital phase with a minimum at orbital phases $\phi=0.6-0.8$ and a maximum just after the primary minimum $\left(f_{2}\right)$ and at $\phi \approx 0.25\left(f_{1}\right)$. The different shape of amplitude modulation of the $f_{1}$ and $f_{2}$ modes points to different $(l, m)$ mode structures. The modulation itself can be explained by assuming a variable extinction due to gas streams and an inhomogeneous accretion annulus that weakens the light from different regions of the primary depending on its orbital position. This assumption is well supported by the gas density distribution obtained in preliminary hydrodynamic simulations.

We found strong variations and cycle-to-cycle variable shapes of the orbital RV-curves of Balmer lines that have maximum magnitude in the $\mathrm{H} \alpha$ line indicating a strong variability of mass-transfer rates and a non-stationary circumbinary envelope.
\end{abstract}

Key words. stars: binaries: eclipsing - stars: variables: general - stars: oscillations - stars: individual: RZ Cas

\section{Introduction}

The pulsation phenomenon in mass-accreting stars in Algoltype systems was recently found to be widely present. The coexistence of mass-accretion and pulsations in the prime components allow for attractive studies of this group of stars using new asteroseismic methods (Mkrtichian et al. 2002b).

The A $3 \mathrm{~V}+\mathrm{K} 0 \mathrm{IV}$ eclipsing binary RZ Cas is an active semi-detached Algol system showing complex features in its light-curve and in radial velocities. The primary minimum is a partial eclipse (Narusawa et al. 1994) and the light-curve is distorted, possibly by star spots (e.g. Maxted et al. 1994) and/or by non-symmetric circumstellar matter (Olson 1982;

Send offprint requests to: $\mathrm{H}$. Lehmann,

e-mail: lehm@tls-tautenburg.de

$\star$ The research is based on spectroscopic observations made with the 2-m telescope at the Thüringer Landessternwarte Tautenburg, Germany.

$\star \star$ Table 3 is only available in electronic form at the CDS via anonymous ftp to cdsarc.u-strasbg.fr $(130.79 .128 .5)$ or via http://cdsweb.u-strasbg.fr/cgi-bin/qcat?J/A+A/413/293
Varricatt et al. 1998). The non-symmetric distribution of circumstellar matter was also suggested by Richards \& Albright (1999) based on the observation of single-peak $\mathrm{H} \alpha$ emission.

Short-period light variability of RZ Cas was reported by different authors (Olson 1982; Edwin \& Gears 1992; Davis \& Balonek 1996). Ohshima et al. $(1998,2001)$ were the first who showed that this variability is caused by oscillations of the primary with a dominant frequency of $64.2 \mathrm{c} \mathrm{d}^{-1}$. Three consecutive multisite campaigns were undertaken in 1999, 2000, and 2001. The dominant oscillation was confirmed during the 1999 (Rodriguez et al. 2002) and 2000 year (Mkrtichian et al. 2003a) campaigns.

In 2001 D.M. organized a multi-site campaign including photometric and spectroscopic observations of RZ Cas to perform a detailed study of the binary orbit, the frequency spectrum of pulsations, and of the effects of accretion and a non-stationary circumstellar envelope. At the same time, 2D and 3D hydrodynamic modeling of mass-transfer in the RZ Cas system were started to interprete the spectroscopic and photometric observations (Mkrtichian et al. 2003a). Here we report the first results of the analysis of the RZ Cas RVs. 
Table 1. Journal of observations. For each run we give the mean HJD $2452100+$, the orbital phase coverage, and the number of obtained spectra.

\begin{tabular}{|rcr|ccr|}
\hline \hline HJD & $\phi$ & $N$ & HJD & $\phi$ & $N$ \\
\hline 83.40 & $0.52 \ldots 0.77$ & 112 & 92.39 & $0.16 \ldots 0.17$ & 7 \\
86.32 & $0.07 \ldots 0.11$ & 9 & 93.44 & $0.87 \ldots 0.25$ & 85 \\
87.50 & $0.90 \ldots 0.22$ & 103 & 94.65 & $0.96 \ldots 0.10$ & 32 \\
88.45 & $0.77 \ldots 0.97$ & 85 & 95.51 & $0.63 \ldots 0.93$ & 142 \\
89.37 & $0.61 \ldots 0.67$ & 27 & 96.50 & $0.38 \ldots 0.77$ & 153 \\
90.55 & $0.52 \ldots 0.72$ & 82 & 97.53 & $0.24 \ldots 0.61$ & 125 \\
\hline
\end{tabular}

\section{Observations and data reduction}

We obtained time series of high-resolution CCD spectra of RZ Cas on 12 nights in October 2001. All spectra were taken with the coudé-echelle-spectrograph of the 2-m telescope of the Thüringer Landessternwarte in Tautenburg. In Table 1 we give the journal of observations. The exposure time per spectrum is $150 \mathrm{~s}$, with a mean sampling time of about $200 \mathrm{~s}$. Spectra cover a wavelength range from 4700 to $7400 \AA$ with a spectral resolution of 40000 .

Data reduction was done using standard MIDAS routines. Reduction included filtering of cosmics, bias and background subtraction, flat fielding, optimum extraction of spectra, wavelength calibration by a Th-Ar lamp, and normalization to the local continuum. Small nightly instrumental shifts were compensated for by using a larger number of telluric $\mathrm{O}_{2}$ lines.

\section{Radial velocity determination}

Metal lines. In some of its lines (Ca I, Fe I) RZ Cas shows contributions of the secondary, whereas for other ions $(\mathrm{Mg} \mathrm{I}$, Fe II, Ti II) no such contribution can be found. Table 2 lists the strongest and less blended lines for both cases. Figure 1 shows the CaI doublet, which shows the strongest contributions of the secondary.

RVs of the primary were measured by a cross-correlation of the RV-rebinned spectra in the range 4970 to $5550 \AA$. This region is free of visible contributions of the secondary and almost free of any telluric absorption features. First RVs were obtained by using one arbitrary spectrum of RZ Cas as the template. After that we shifted, according to the obtained RVs, about 40 spectra centered around the orbital phase of secondary minimum to laboratory wavelengths and co-added the spectra. The co-added spectrum served as an improved template for the next step of cross-correlation. The use of spectra near to secondary minimum should additionally minimize the influence of the secondary. The finally obtained mean accuracy in RV was of $0.11 \mathrm{~km} \mathrm{~s}^{-1}$. This accuracy, obtained from cross-correlation, is about four times higher than that obtained from a RV determination by Gaussian line fits.

RVs of the secondary were measured from the CaI and Fe I lines listed in the right column of Table 2. Here we fitted two Gaussians to the observed line profiles, one for the primary and one for the secondary. This procedure works only outside the primary eclipse, of course. The obtained mean accuracy is of $1.7 \mathrm{~km} \mathrm{~s}^{-1}$.
Table 2. Spectral lines having their origin mainly in the primary and spectral lines with the strongest contributions of the secondary (wavelength in $\AA$, ions, and RMT number of transition).

\begin{tabular}{|rrr|rrr|}
\hline \hline \multicolumn{3}{|c|}{ primary } & \multicolumn{3}{|c|}{ secondary } \\
\hline 5183.604 & $\mathrm{Mg}_{\text {I }}$ & 2 & 6102.722 & $\mathrm{Ca} \mathrm{I}$ & 3 \\
5528.398 & $\mathrm{Mg}_{\text {I }}$ & 9 & 6122.219 & $\mathrm{Ca} \mathrm{I}$ & 3 \\
5275.994 & Fe II & 49 & 4957.453 & Fe I & 318 \\
5284.092 & Fe II & 41 & 5615.652 & Fe I & 686 \\
5316,693 & Fe II & $48+49$ & 5658.684 & Fe I & 685 \\
4911.205 & Ti II & 114 & & & \\
5226.534 & Ti II & 70 & & & \\
\hline
\end{tabular}

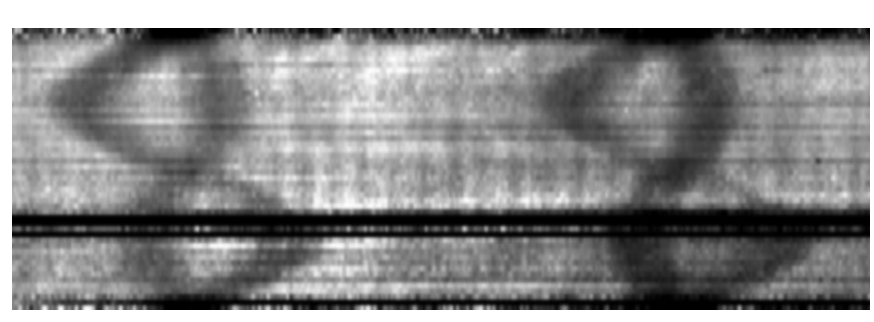

Fig. 1. The Ca I doublet $6103 / 6122 \AA$ shows the strongest absorption lines of the secondary. We show all spectra, binned in vertical direction into 50 phase bins folded with the orbital period. The lower and upper edges correspond to the phase of primary minimum.

Hydrogen lines. The observed $\mathrm{H} \alpha$ line profile of RZ Cas is a superposition of a strong absorption and a weak, variable, single-line emission component (Richards \& Albright 1999). In this paper, devoted to an improved orbital solution and the search for pulsations, we do not attempted to extract these contributions. The radial velocities of $\mathrm{H} \alpha$ and $\mathrm{H} \beta$ were measured by fitting the line profiles by a superposition of two Gaussian and one Cauchy profiles with common line centers and variable line depths and widths. One of the Gaussians was used to fit the line center and the Cauchy profile to fit the line wings. The second Gaussian was used for the intermediate part of the profiles. All RVs are listed in Table $3^{1}$.

\section{Orbital solutions}

Figure 2 shows an orbital phase diagram of the RVs determined from the metal lines of the primary (Table 2, left column) and from the $\mathrm{H} \alpha$ and $\mathrm{H} \beta$ lines. Whereas the phase diagram for the metal lines looks very smooth, the hydrogen lines RVs show a large scatter. At a first sight, the deviations from the expected Keplerian orbital curve seem to be in phase with the orbit for the metal lines, and they show clearly cycle-to-cycle variable differences for the hydrogen lines. The behaviour of the hydrogen lines will be discussed in more detail in Sect. 7. In the following, to derive the orbital solution, we will only consider the metal lines RVs.

1 Table 3 is only available in electronic form at the CDS via anonymous ftp to cdsarc.u-strasbg.fr (130.79.128.5) or via http: //cdsweb.u-strasbg.fr/cgi-bin/qcat? J/A+A/413/293. 


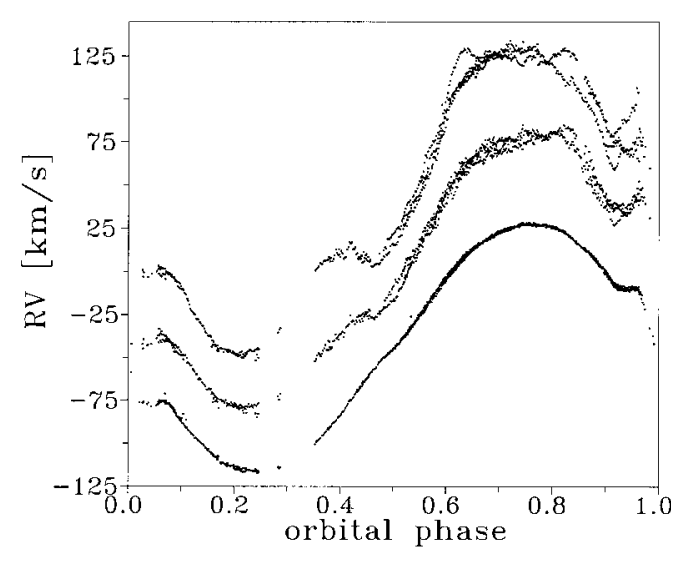

Fig. 2. RVs of metal and hydrogen lines folded with the orbital period of 1.1952572 . The $\mathrm{H} \beta$ and $\mathrm{H} \alpha$ lines RVs are displaced against the metal lines RVs by $+40 \mathrm{~km} \mathrm{~s}^{-1}$ and $+80 \mathrm{~km} \mathrm{~s}^{-1}$, respectively.

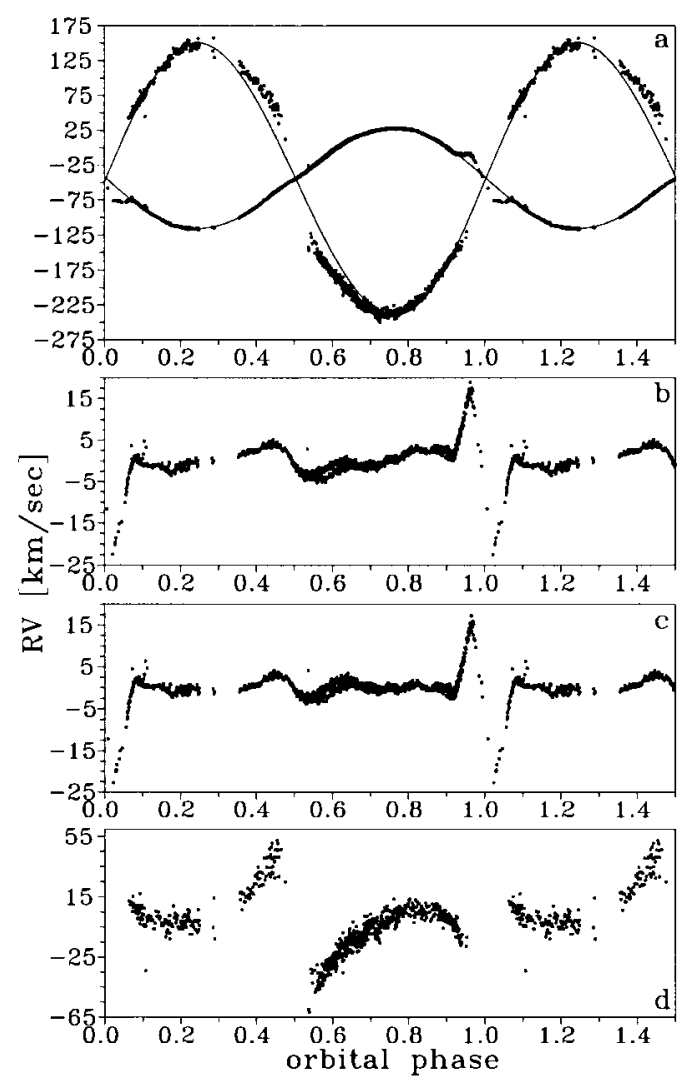

Fig. 3. Phase diagrams folded with the orbital period. Phase zero corresponds to Min I. a) RVs and orbital curves of primary and secondary component according to orbital solution II. b) Residuals of the primary after subtracting solution I and c) after subtracting solution II. d) Residuals of the secondary after subtracting solution II.

Our calculation of orbit is based on the method of differential corrections. We started with the orbital ephemeries derived by Narusawa et al. (1994) from the RZ Cas light curves:

Min I = HJD $2448960.2122+1.1952572 E$

and computed a first orbital phase diagram like shown in Figs. 2 and 3a. From these figures it can be clearly seen that the orbital curve of both components is highly distorted by the rotation effect during the eclipses. As expected, the maximum distortion
Table 4. Orbital solutions of RZCas assuming a circular orbit (solution I) as well as a non-circular orbit (solution II). The orbital period is fixed to $P=1.1952572$. $T_{\mathrm{P}}$ is the epoch of periastron passage, $T_{1}$ corresponds to Min I, epochs are given as HJD 2452 100+. For comparison we list also the elements obtained by Duerbeck \& Hänel (1978) from metal lines.

\begin{tabular}{lrrrl}
\hline \hline & Duerbeck & solution I & solution II & \\
\hline$K_{1}$ & $70.5(1.4)$ & $71.36(12)$ & $71.691(76)$ & $\mathrm{km} \mathrm{s}^{-1}$ \\
$\gamma_{1}$ & $-45.5(1.1)$ & $-44.035(95)$ & $-43.946(54)$ & $\mathrm{km} \mathrm{s}^{-1}$ \\
$K_{2}$ & & $-200.78(34)$ & $-194.88(67)$ & $\mathrm{km} \mathrm{s}^{-1}$ \\
$\gamma_{2}$ & & $-44.42(21)$ & $-44.56(51)$ & $\mathrm{km} \mathrm{s}^{-1}$ \\
$T_{1}$ & & $93.3872(57)$ & $93.388(15)$ & $\left(\right.$ from $\left.T_{\mathrm{P}}\right)$ \\
$T_{\mathrm{P}}$ & & & $93.3857(80)$ & \\
$\mathrm{e}$ & $0.024(23)$ & & $0.0288(12)$ & \\
$\omega$ & $87(2)$ & & $89.2(2.4)$ & $\mathrm{deg}$ \\
\hline
\end{tabular}

occurs for the RVs of the primary and of the secondary at primary (Min I) and secondary minimum (Min II), respectively. RVs of the primary are slightly distorted also at secondary minimum, however.

For the orbital calculation we used only RVs of the primary outside the primary eclipse with orbital phase positions between 0.1 and 0.9 and RVs of the secondary at phases outside the secondary eclipse with phase positions outside 0.35 to 0.65 . In a first step we assumed a circular orbit. Since the time basis of our observations is too small to derive the orbital period with an accuracy comparable to that derived from the light curves, we fixed the period to Narusawa's value of 1.1952572. Table 4 lists the orbital elements obtained from a combined solution for both components as solution I (RVs were weighted according to their individual errors of measurement). The corresponding orbital curves are shown in Fig. 3 a.

Figure $3 \mathrm{~b}$ shows the residuals after subtracting orbital solution I from the RVs of the primary. There are strong deviations during primary minimum. The amplitudes of the RV distortion before and after Min I are clearly different.

The rotation effect in eclipsing binaries is well-known since a long time. The probable existence of a measurable effect due to the rotation of eclipsing stars was recognized by Schlesinger already in 1909, during an investigation of the orbital RVs of $\delta \mathrm{Lib}$ and $\lambda$ Tau. Rossiter's (1924) isolation of the rotation effect in $\beta$ Lyr was the beginning of the recognition of it as a means of determining the dimensions of eclipsing systems. The - very rarely observed - asymmetric rotation effect was first found by McLaughlin (1937) in Algol. McLaughlin tried to explain the observed asymmetry be line blending due to a third body in the Algol system. We will discuss the observed asymmetry of the rotation effect in RZ Cas in Sect. 7.

It can be seen from Fig. $3 b$ that there is a slope in the RVs between the two minima which could be due to a noncircularity of the orbit. To check this we allowed for non-zero eccentricity and obtained the orbital elements listed in Table 4 as solution II. One difference between the phase diagrams resulting from solutions I and II is directly shown from the residuals of the primary: There is now a flat course between the two 
Table 5. Masses of the components derived from orbital solution I.

\begin{tabular}{ccc}
\hline \hline$M_{1} \sin ^{3} i$ & $M_{2} \sin ^{3} i$ & $q=M_{2} / M_{1}$ \\
$1.84 \pm 0.02 M_{\odot}$ & $0.654 \pm 0.006 M_{\odot}$ & $0.355 \pm 0.001$ \\
\hline
\end{tabular}

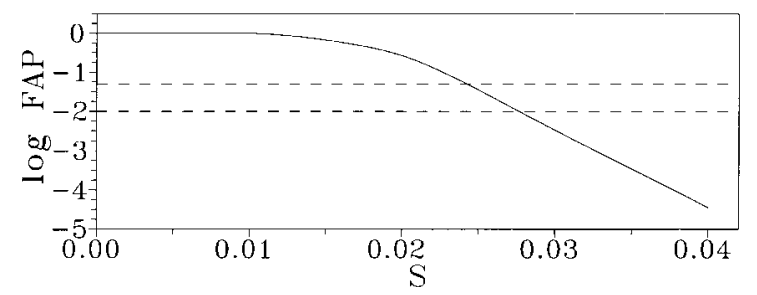

Fig. 4. Empirical false-alarm probability distribution. The upper dashed line indicates the 5\% FAP limit $(S=0.0241)$, the lower one the $1 \%$ FAP limit $(S=0.0275)$.

minima (Fig. 3c) which means that the RV extrema are slightly better fitted than in the circular approximation.

Spectroscopic orbits of RZ Cas have been determined by Horak (1952) and by Duerbeck \& Hänel (1978) based on photographic spectra and by Maxted et al. (1994) based on Reticon spectra. Only Duerbeck \& Hänel (1978) derived a more or less significant value of the eccentricity of a possibly non-circular orbit. Despite the much lower data accuracy their results obtained from the metallic lines (see Table 4) are in very good agreement with our results. Nevertheless, the small eccentricity is in doubt. Although its value of 0.029 is significant with respect to the computed error, we have to take care of all possibly distorting effects like envelope contributions, transit effects, surface spots, and pulsations which are expected to be present in RZ Cas. These effects can cause much of the variations seen in the orbital residuals and also mimic an eccentricity of such a small amount.

Thus, we use solution I to derive preliminary masses of the components. Solution II was only used to obtain a better fitting to build the residuals for a search for short-term RV variations. Table 5 lists the masses of the components obtained from solution I. These values are not corrected for non-Keplerian effects due to the ellipticity of the components. The correction will be done in a forthcoming paper, after a more complete modeling of the RZ Cas system has been made.

\section{Search for short-term periods}

For the period search we used a modified Scargle diagram (see Lehmann et al. 1999) in which the ordinate $S$ gives the reduction in the sum of squares. It is $S=1-\sigma / \sigma_{0}$, where $\sigma$ is the variance of the residuals of a sine-wave fit and $\sigma_{0}$ is the total variance of the data. To estimate the false-alarm probability (FAP), i.e. the probability that a peak of a certain height observed in the periodogram results from pure noise, we computed an empirical FAP distribution based on the time sampling of our data and assuming Gaussian distributed noise with $\sigma=0.11 \mathrm{~km} \mathrm{~s}^{-1}$. The resulting FAP distribution shown in Fig. 4 is based on 20000 trials in a frequency range from 0 to $100 \mathrm{c} / \mathrm{d}$ with a resolution of 25000 frequencies per trial.

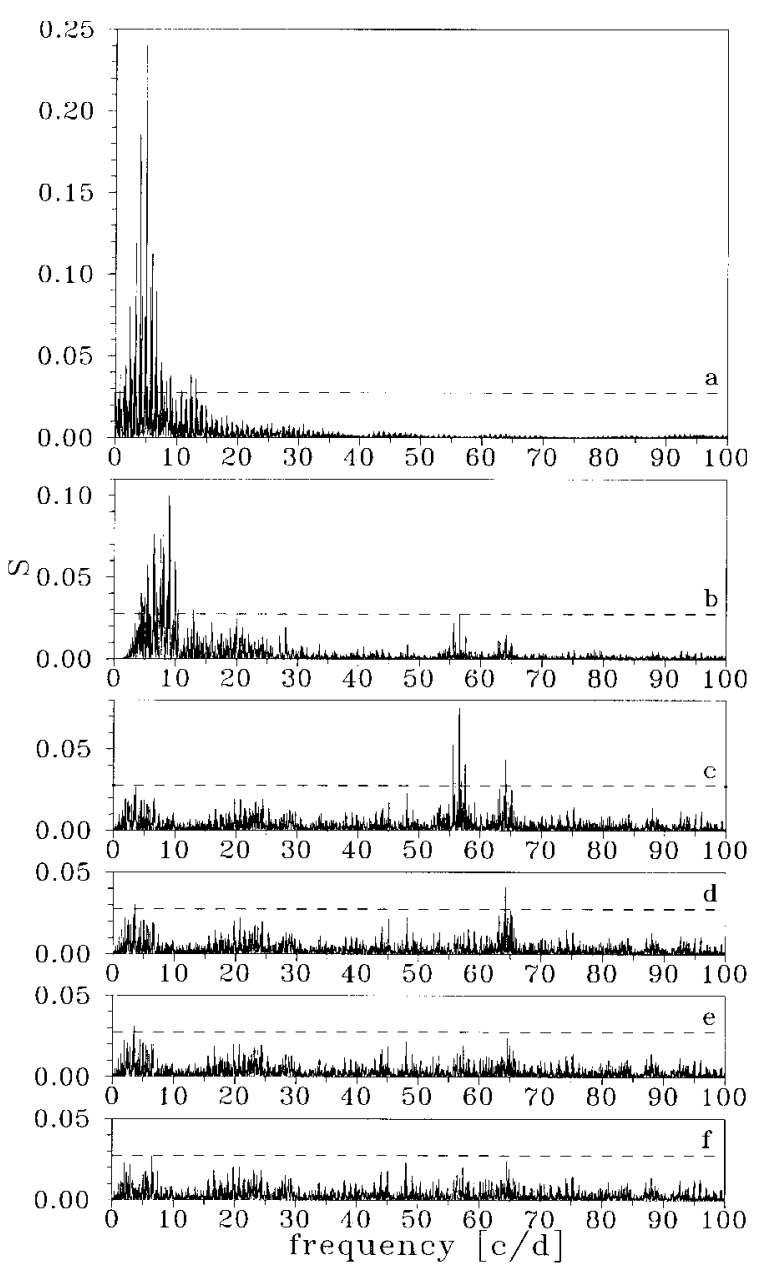

Fig. 5. Periodograms obtained after a) subtracting orbital solution II, b) subtracting a high degree polynomial from all data and lowdegree polynomials from the individual data sets, c) pre-whitening for all low-frequency distributions up to a limit of $25 \mathrm{~cd}^{-1}$, d) prewhitening for $f_{1}=56.600 \mathrm{c} \mathrm{d}^{-1}$, e) for $f_{2}=64.189 \mathrm{c} \mathrm{d}^{-1}$, and f) for the $0.453 \mathrm{c} \mathrm{d}^{-1}$ frequency. The dashed lines indicate the $1 \%$ FAP limit.

Since we concentrate only on the high-frequency RV variations due to possible pulsations of the primary we tried to eliminate all low-frequency contributions, which are related to fractions of the time scale of rotation (surface spots) and to more or less spontaneous changes due to circumstellar activity in the Algol system. First we subtracted orbital solution II from the data. Figure 5a shows the periodogram of the residuals of the RVs of the primary. In a next step we cleaned the orbital curve by a high-degree polynomial in the RV - orbital phase plane. This procedure also fits the transit effects. Then each individual run was cleaned up by a low degree polynomial. The periodogram obtained at this stage is shown in Fig. 5b.

In the last step we pre-whitened each data set for all lowfrequency distributions up to a limit of $25 \mathrm{~cd}^{-1}$ and merged the residuals of each run together for a further period search in the entire data set. Figure $5 \mathrm{c}$ to $\mathrm{f}$ show the periodograms at this stage and as they were obtained during further steps of pre-whitening. First we find $f_{1}=56.6 \mathrm{c} / \mathrm{d}$, followed by $f_{2}=64.2 \mathrm{c} / \mathrm{d}$. The peak heights of both periods are distinctly above the empirical 1\% FAP limit. The coincidence 
Table 6. Short-term frequencies found by successive pre-whitening. We list frequency, period, and semi-amplitude as well as the peak height $S$ in the periodograms. Errors are given in parentheses in units of the last digit.

\begin{tabular}{ccccc}
\hline \hline & $f\left[\mathrm{c} \mathrm{d}^{-1}\right]$ & $P[\mathrm{~d}]$ & $K\left[\mathrm{~m} \mathrm{~s}^{-1}\right]$ & $S$ \\
\hline$f_{1}$ & $56.600(4)$ & $0.017668(1)$ & $151(27)$ & 0.077 \\
$f_{2}$ & $64.189(6)$ & $0.015579(1)$ & $99(26)$ & 0.041 \\
\hline
\end{tabular}

Table 7. High-frequency peaks found within single nights. For every run we give JD as $2452100+$, mean orbital phase $\phi$, frequencies in $\mathrm{cd}^{-1}$ and corresponding amplitudes in $\mathrm{m} \mathrm{s}^{-1} \cdot f_{1}$ and $f_{2}$ mean frequencies found very near to the frequencies $f_{1}$ and $f_{2}$ obtained from the entire data set, additional frequencies found in the high-frequency domain are listed as $f_{a}$. Errors are given in parenthesis in units of the last digit.

\begin{tabular}{cccccccc}
\hline \hline JD & $\phi$ & $f_{1}$ & $K_{1}$ & $f_{2}$ & $K_{2}$ & $f_{a}$ & $K_{a}$ \\
\hline 83 & 0.64 & $55.5(6)$ & $160(63)$ & $62.5(6)$ & $145(60)$ & & \\
87 & 0.08 & $57.1(4)$ & $291(87)$ & $64.1(3)$ & $388(81)$ & & \\
88 & 0.87 & $57.6(11)$ & $117(75)$ & $63.2(9)$ & $130(74)$ & $68.9(8)$ & $165(74)$ \\
90 & 0.63 & $57.1(7)$ & $173(51)$ & $63.1(8)$ & $151(67)$ & $48.4(7)$ & $169(67)$ \\
93 & 0.04 & $56.6(2)$ & $339(76)$ & $64.7(5)$ & $235(79)$ & & \\
95 & 0.78 & & & $64.7(4)$ & $177(61)$ & & \\
96 & 0.61 & $55.9(4)$ & $153(62)$ & & & & \\
97 & 0.47 & $56.0(4)$ & $199(70)$ & & & & \\
\hline
\end{tabular}

of $f_{2}$ (smaller peak height) with the value of the frequency known from photometry additionally confirms the significance of the peaks. After subtracting both contributions from the data we find only one frequency with a peak height exceeding the 1\% FAP limit (Fig. 5e). This peak lies in the low-frequency range $\left(f_{3}=0.453 \mathrm{c} \mathrm{d}^{-1}\right)$, however, and has no physical meaning. The periodogram of the residuals after subtracting also the $f_{3}$-variation shows no more peaks above the $1 \%$ FAP limit (Fig. 5f). The final $\sigma$ after removing $f_{1}$ to $f_{3}$ is $390 \mathrm{~m} \mathrm{~s}^{-1}$. Table 6 lists the results. The errors of frequencies and amplitudes were estimated by applying the method of differential corrections using $f_{1}$ and $f_{2}$ as obtained from the periodograms as starting values.

A similar period search was done where only out-of-eclipse orbital phases were taken into account. Although we expected cleaner periodograms with even more distinct $f_{1,2}$ peaks, the obtained results are comparable to that shown in Fig. 5 but the $f_{1,2}$ peaks were less pronounced. A probable reason might be the fact that we find the largest amplitudes of $f_{1,2}$ very soon after the primary eclipse, as described in the next section.

The period search in the hydrogen lines was done in a similar way. Analysis showed, however, that low frequency contributions, instabilities and noise are much higher than for the metal lines. Only in the $\mathrm{H} \alpha \mathrm{RVs}$ and only in some single runs are the frequencies $f_{1}$ and $f_{2}$ spuriously present. We could not detect any signal in the $\mathrm{H} \beta \mathrm{RVs}$.

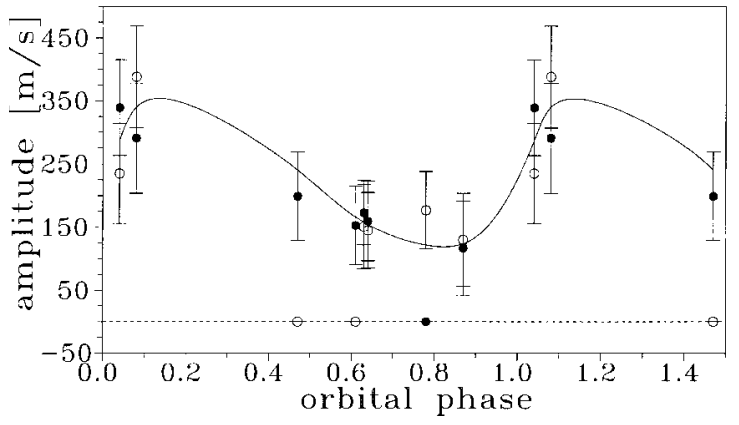

Fig. 6. Phase diagram of the amplitudes of $f_{1}$ (filled circles) and $f_{2}$ (open circles) folded with the orbital period. Phase zero corresponds to Min I. The points shown at the zero-level line correspond to nights where no corresponding peaks could be detected. The solid curve shows a cubic spline through the mean of the amplitudes of all pairs $\left(f_{1}, f_{2}\right)$.

\section{Amplitude modulation}

From an inspection of the metal lines RVs of single runs we found that the contributions of frequencies $f_{1}$ and $f_{2}$ are of different strength on different nights of observation. To check for a possible periodicity in the amplitude variation we investigated each data set separately. We pre-whitened the RVs of each single night for all low-frequency contributions until the largest peak in the periodogram occurred at a frequency above $30 \mathrm{~cd}^{-1}$. Then we determined the frequencies and corresponding amplitudes of each larger peak above $30 \mathrm{~cd}^{-1}$. Table 7 lists the results. In any but two nights, where a third frequency occurs and we observe a triplet, the largest peak in the periodograms corresponds either to $f_{1}$ or $f_{2}$. From the amplitudes of the contributions near to $f_{1}$ and $f_{2}$ we built the phase diagram shown in Fig. 6. Here the HJDs of the single runs are folded with the orbital period.

From Fig. 6 we conclude that the modulation of amplitudes is related to the time scale of orbital motion. To verify this we built a multiple frequency model where we included $f_{1}, f_{2}$, and the terms $f_{1} \pm \Delta f_{1}$ and $f_{2} \pm \Delta f_{2}$. Then we fitted this model to the entire data set (after cleaning for all low-frequency contributions), in the sense of a least squares fit with variables $\Delta f_{1,2}$. For both pulsation frequencies, the best fit was obtained for a frequency splitting of $\Delta f_{1} \approx \Delta f_{2} \approx 1.2$, which is the orbital period.

A more detailed behaviour of the $f_{1}$ and $f_{2}$ variations can be observed in Fig. 7 where we divided all RVs into orbital phase bins of $\Delta \phi=0.25$. The resulting amplitudes confirm the amplitude modulation with the orbital period. Moreover, a phase shift between the $f 1$ and $f_{2}$ amplitudes can be clearly seen. Whereas the $f_{2}$ variation shows the largest amplitudes at orbital phase 0.1 , the $f_{1}$ variation is largest at $\phi \approx 0.3$. The minima of the $f_{1}$ and $f_{2}$ amplitudes show the same phase shift of about 0.2 .

\section{Discussion and conclusion}

The investigation of the newly obtained high-resolution spectroscopic time series of RZ Cas led to a very precise determination of the orbital elements and to the first spectroscopic detection of rapid pulsations in an Algol system, with two dominant 


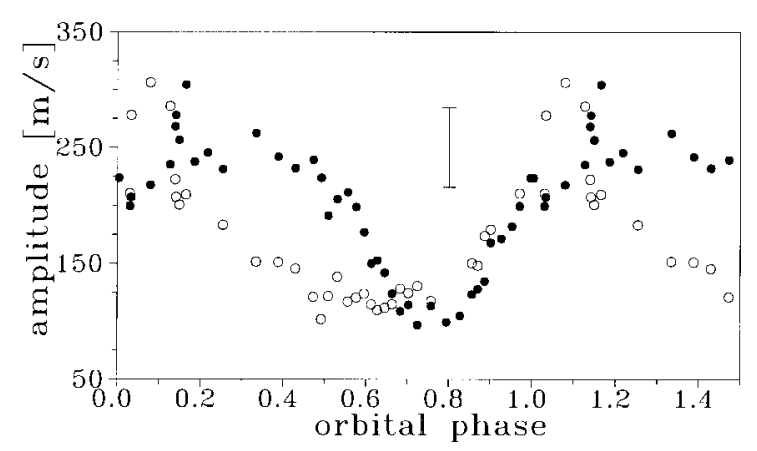

Fig. 7. As Fig. 6, but amplitudes derived within orbital phase bins. The typical error in amplitude is indicated by the single error bar.

frequencies of 56.6 and $64.2 \mathrm{~cd}^{-1}$ - both in agreement with the values obtained from a simultaneous photometric campaign (Mkrtichian et al. 2003b).

Whereas from 1997 to October 2000 RZ Cas showed dominant monoperiodic photometric oscillations with a frequency of $64.2 \mathrm{c} \mathrm{d}^{-1}$ and a semi-amplitude of about $9 \mathrm{mmag}$ (Ohshima et al. 1998, 2001; Rodriguez et al. 2002; Mkrtichian et al. 2002a), the spectrum of rapid oscillations became multiperiodic in 2001 and the photometric amplitude of the former dominant mode decreased by one order of magnitude (Mkrtichian et al. 2003a). This is the first observational evidence of abrupt changes in the pulsation amplitude of the massaccreting component of an Algol system.

We find the RV amplitude of both pulsation modes to be variable over the orbital period with a maximum just after Min I $(\phi \approx 0.1)$ and a broad minimum at orbital phases $\phi=0.6-0.9$. This amplitude modulation can be explained by assuming variable extinction due to a non-symmetric gas-envelope and gasstreams that weaken the light from different surface regions of the pulsating primary in dependence on its orbital position. First results of 2D and 3D hydrodynamical calculations for the RZ Cas system (Nazarenko \& Mkrtichian 2003; Mkrtichian et al. 2003a) show the formation of an asymmetric gas envelope in the orbital plane around the gainer.

The line-of-sight envelope column density in the equatorial plane is highest at orbital phases just before Min I. In this case the gas-stream and the most dense part of the envelope is projected against the surface of the pulsator. It is lowest at phases just after Min I. Figure 8 illustrates these conditions for different viewing angles. It was obtained from preliminary $2 \mathrm{D}$ calculations of the RZ Cas system. A confirmation of the amplitude variation by the photometric data is important. A detailed study will be presented in a forthcoming paper (Mkrtichian et al. 2003b).

The difference in the shape of amplitude modulation between both modes might indicate their different structure (i.e. different $(l, m)$ quantum numbers): The surface velocity fields of both modes are attenuated by the inhomogeneous gas envelope; their different spatial structure gives rise to different diskintegrated amplitudes, however. For instance, for a sectorial $(l=|m|)$ mode that possesses maximum pulsation amplitudes on the stellar equator, we would expect a clear anti-correlation between observed pulsation amplitudes and the radial column

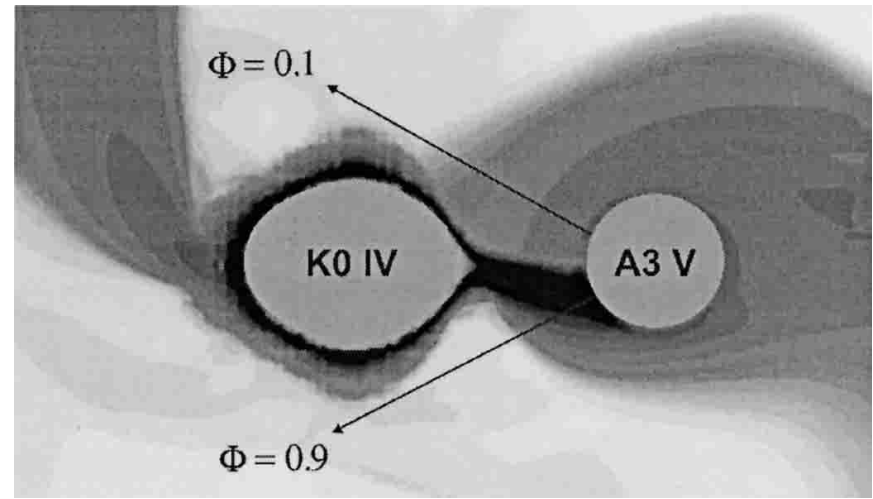

Fig. 8. Schematic drawing of the RZ Cas system viewed at different orbital positions.

density distribution of circumbinary matter. Such an analysis will be done in the framework of the hydrodynamical modeling of the RZ Cas system.

The hypothesis of an inhomogeneous accretion annulus whose density varies with orbital phase is also in agreement with the observed asymmetric rotation effect. On the descending branch of Min I, the secondary star is screening the lefthand semi-sphere which moves towards the observer. At the same time, the atmosphere in the right-hand semi-sphere is shadowed by the dense and cool gas-stream and envelope cloud what reduces its contribution to the positive RV jump. On the ascending branch of Min I, the left-hand semi-sphere, moving towards the observer, is seen through a thin gas envelope while the right-hand semi-sphere is screened by the secondary star. This gives rise to a larger amplitude of the negative RV jump compared to the positive one.

For completeness, we will discuss two additional hypotheses. First, the observed asymmetry of the rotation effect can also arise if the rotation axis of the primary is inclined to the orbital plane. This scenario, however, is in contradiction to the close binary system with its short orbital period: For RZ Cas the circularization and synchronization effects work on a relatively short time scale and a large inclination of the rotation axis seems to be very unlikely.

The second hypothesis assumes the rotation axis to be perpendicular to the orbital plane but the pulsation axis is inclined, which would also give rise to an amplitude modulation of pulsation modes. Such oblique pulsations in close binaries were predicted by Shibahashi (2002) for the special case that the eigenmode is deformed to have a quadrupole component whose symmetric axis coincides with the direction of the companion star.

The gas-envelope screening scenario, on the other hand, explains both the rotation effect and the amplitude modulation of pulsation. The final answer, whether the inhomogeneous gasenvelope effect or an inclination of pulsation axis (or both) are working in RZ Cas will be found only after the detailed modeling of modulation effect and envelope's spatial filter effects of non-radial pulsation modes using the data of 3D hydrodynamic simulations.

A comparison between Balmer lines and weak metal lines shows strong and cycle-to-cycle variable differences and 
velocity shifts. The observed instability is largest for $\mathrm{H} \alpha$ reflecting the larger influence of the non-stationary circumbinary envelope on the $\mathrm{H} \alpha$ line profile. The $\mathrm{H} \alpha, \mathrm{H} \beta$, and weak metal line RV-curves coincide just after Min I, between orbital phases $0.07-0.12$ (where the line-of-sight density of the circumbinary envelope drops to minimum); at all other phases we see a superposition of different transfer-related processes like gas-streams and changing envelope projection effects that are variable in time. This signals strong non-stationarities in masstransfer rate, gas-stream, envelope structure, temperature, density, and velocity field distributions around the binary system.

A qualitative interpretation of these contributions requires an accurate modeling of the observed profiles including knowledge of the complex physics of the atmosphere-gas-stream-envelope interaction as well as of the emission and absorption line contributions from different parts of the circumbinary envelope. This modeling is impossible without realistic 3D hydrodynamic simulations of mass transfer and the problem is still far from beeing solved. At the present time, we have also no definite explanation for the slight but clearly visible distortion of the RVs of the primary during secondary eclipse (Fig. 3). Since this distortion is strongest for the hydrogen lines (Fig. 2) we can at least exclude possible contributions of secondary lines in the RVs of the primary as the main source.

The present study, which concentrates on the RV variations, is part of a more extended investigation of RZ Cas which will combine the actual results with those from the analysis of simultaneously obtained photometry, with a detailed study of line profile variability, with an abundance analysis, and with a $2 \mathrm{D}$ and 3D hydrodynamic modeling of the RZ Cas system. The final model should be able to explain also the fine-structure of the observed RV variations including the asymmetric rotation effect as well as the low-frequency distortions of the orbital curve which were not examined in the context of the present paper.
Acknowledgements. D.M. acknowledges his work as part of research activities of the Astrophysical Research Center of the Structure and Evolution of the Cosmos (ARCSEC) which is supported by the Korean Science \& Engineering Foundation. A part of his work was funded in 2002 by the German Academic Exchange Service (DAAD).

\section{References}

Davis, S. M., \& Balonek, T. J. 1996, BAAS, 28, 1375

Duerbeck, H. W., \& Hänel, A. 1978, A\&AS, 38, 155

Edwin, P. R., \& Gears, R. T. 1992, PASP, 104, 1234

Horak, H. G. 1952, ApJ, 115, 61

Lehmann, H., Scholz, G., Hildebrandt, G., \& Panov, K. 1999, A\&A, 351,267

Maxted, P. F. L., Hill, G., \& Hilditch, R. W. 1994, A\&A, 282, 821

McLaughlin, D. B. 1937, Publ. Obs. of the Univ. of Michigan, 6, 3

Mkrtichian, D. E., Kusakin, A. V., Gamarova, A. Yu., et al. 2002a, PASPC, 256, 259

Mkrtichian, D. E., Kusakin, A. V., Gamarova, A. Yu., \& Nazarenko, V. 2002b, PASPC, 259, 96

Mkrtichian, D. E., Nazarenko, V., Gamarova, A. Yu., et al. 2003a, PASPC, 292, 113

Mkrtichian, D. E., et al. 2003b, in prep.

Narusawa, S. Y., Nakamura, Y., \& Yamasaki, A. 1994, AJ, 107, 1141

Nazarenko, V., \& Mkrtichian, D. E. 2003, in prep.

Ohshima, O., Narusawa, S., Akazawa, H., et al. 1998, IBVS, 4581

Ohshima, O., Narusawa, S.-Y., Akazawa, H., et al. 2001, AJ, 122, 418

Olson, E. C. 1982, ApJ, 259, 702

Richards, M. T., \& Albright, G. E. 1999, ApJS, 123, 537

Rodriguez, E., Costa, V., Lopez-Gonzalez, M. J., et al. 2002, PASPC, 259, 102

Rossiter, R. A. 1924, ApJ, 60, 15

Schlesinger, F. 1909, Publ. Allegheny Obs. 1, 134 and 3, 28

Shibahashi, H. 2002, PASPC, 259, 82

Varricatt, W. P., Ashok, N. M., \& Chandrasekhar, T. 1998, AJ, 116, 1447 